\title{
Establishment and Validation of a Preoperative MRI-based Nomogram for Predicting the Risk of Malignancy in Patients with Breast Tumor
}

\author{
Jianguo Lai ${ }^{1,4,6 \dagger}$, Jinjiang Lin²†, Hongli Wang ${ }^{3,4 \dagger}$, Yi Sun ${ }^{1,4}$, Yudong Li1 ${ }^{1,4}$, Huan Tian ${ }^{1,4}$, Shiyu Shen ${ }^{3,4}$, \\ Cui Tan ${ }^{4,5}$, Huanhuan Liu ${ }^{1,4,7}$, Fengyan $\mathrm{Yu}^{1,4} \bowtie$ \\ 1. Department of Breast Surgery, Breast Tumor Center, Sun Yat-Sen Memorial Hospital, Sun Yat-Sen University, Guangzhou, Guangdong, China. \\ 2. Department of Radiology, The First Affiliated Hospital of Sun Yat-Sen University, Guangzhou, Guangdong, China. \\ 3. Diagnostic Department, Breast Tumor Center, Sun Yat-Sen Memorial Hospital, Sun Yat-Sen University, Guangzhou, Guangdong, China. \\ 4. Guangdong Provincial Key Laboratory of Malignant Tumor Epigenetics and Gene Regulation, Sun Yat-Sen Memorial Hospital, Sun Yat-Sen University, Guangzhou, \\ Guangdong, China. \\ 5. Department of Pathology, Sun Yat-Sen Memorial Hospital, Sun Yat-Sen University, Guangzhou, Guangdong, China. \\ 6. Department of Breast Cancer, Cancer Center, Guangdong Provincial People's Hospital and Guangdong Academy of Medical Science, Guangzhou, Guangdong, China. \\ 7. Department of Plastic Surgery, Sun Yat-sen Memorial Hospital, Sun Yat-sen University, Guangzhou, Guangdong, China.
}

†These authors contributed equally to this study.

$\square$ Corresponding author: Dr. Fengyan Yu, Breast Tumor Center, Sun Yat-Sen Memorial Hospital, Sun Yat-Sen University, 107 Yanjiang West Road, Guangzhou, 510120, P. R. China. Tel:+0086-13570982163; Fax:+0086-020-34071287; E-mail: fengyanyu_summer@163.com OR yufengy@mail.sysu.edu.cn

(c) The author(s). This is an open access article distributed under the terms of the Creative Commons Attribution License (https://creativecommons.org/licenses/by/4.0/). See http://ivyspring.com/terms for full terms and conditions.

Received: 2020.06.13; Accepted: 2020.09.20; Published: 2021.01.01

\begin{abstract}
Purpose: To establish a preoperative nomogram incorporating morphological and dynamic contrast-enhanced (DCE) features to individually predict the risk of malignancy in patients with breast tumor.

Methods A total of 447 consecutive female patients who were divided into the primary cohort $(n=326)$ and the validation cohort $(n=121)$ were enrolled between March 2015 to January 2018. Univariate and multivariate logistic regression analyses were used to identify the potential independent indicators of malignancy. An MRI-based nomogram integrating morphological features and kinetic curves was developed to achieve individualized risk prediction of malignancy in patients with breast masses. The discrimination, calibration ability and clinical utility of the MRI-based model were assessed using C-index, calibration curve and decision curve analysis.

Results: Age, tumor size, margin, internal enhancement characteristics, and kinetic curve were confirmed as the independent predictors of malignancy. The AUC of MRI-based nomogram was 0.940 (95\% Cl: $0.911-0.970)$ and 0.894 (95\% Cl: 0.816-0.974) in the primary cohort and validation cohort, respectively. 447 patients were subdivided into the low-risk group $(n=107)$ and high-risk group $(n=340)$ based on the optimal cut-off value of 21.704. The high-risk patients had a higher likelihood of harboring malignancy.

Conclusion: The MRI-based nomogram can be used to achieve an accurate individualized risk prediction of malignancy and reduce unnecessary breast biopsy.
\end{abstract}

Key words: Breast; Nomogram; Magnetic resonance imaging; Diagnosis

\section{Introduction}

Breast cancer is the most common cancer diagnosed and is the second leading cause of cancer death among women. Breast cancer survival varies substantially by stage at diagnosis, early diagnosis is vital for the prognosis of breast cancer [1]. Breast magnetic resonance imaging (MRI) is considered as a crucial imaging tool for diagnosis and preoperative staging of breast cancer [2]. And its clinical importance has gradually increased in recent years. Breast MRI has even changed the clinical 
management for breast cancer.

Besides mammography, ultrasound, positron emission tomography (PET) and MRI are often used as alternate modalities, the advantages of MRI over other modalities are to describe the tumor in dense breast tissue and cancer in a three-dimensional way [3-7]. Another advantage of MRI is the high sensitivity $(>90 \%)$ in breast cancer diagnosis [8]. Thus, MRI has the potential to facilitate the diagnosis in patients with breast tumor. However, the specificity of MRI is relatively low. A false-positive result may trigger severe anxiety, unnecessary breast biopsies, and overtreatment. The relatively low specificity of MRI has been attributed to overlapping features in benign and malignant breast tumors $[9,10]$. And the methods of estimating the MRI findings have been somewhat intuitive and relatively inter-observer variability [11]. On this account, the American College of Radiology (ACR) issued and updated the Breast Imaging Reporting and Data System (BI-RADS) MRI lexicon which standardized the terminology of morphological features and kinetic curve of breast tumors [12]. Even so, BI-RADS didn't identify the correlation between tumor characteristics and malignancy risk. According to the morphological and dynamic contrast-enhanced (DCE) features, the conventional diagnosis of breast tumor on MRI is mainly based on the experience of radiologists. there was not a predictive tool to evaluate the individual risk of malignant tumor based on breast MRI.

In view of these reasons, the following hypothesis immediately puts forward: whether a predictive tool is constructed to evaluate the individual risk of malignancy in patients with breast tumor based on morphological and DCE features?

Recently, nomogram which creates a simple graphical representation of a predictive statistical model [13], has become widely used as predictive tools for diagnosing malignancies. Briefly, A nomogram, which is a user-friendly pictorial representation of mathematical tool, could be applied to predict a numerical probability of an event for an individual patient[14].

Therefore, the purpose of this study was to establish a preoperative nomogram incorporating morphological and DCE features to individually predict the risk of malignancy in patients with breast tumor.

\section{Methods}

\section{Study Population}

A total of 447 consecutive female patients who were suspected to have solid breast tumor (BI-RADS 4 and 5) on MRI examination were enrolled from March
2015 to January 2018. Our institutional review board approved this retrospective study and waived the requirement for informed consent. The inclusion criteria were as follows: 1) age $>20$; 2) underwent breast biopsy or surgical excision; 3) underwent MRI examination; The exclusion criteria were as follows: 1 ) underwent neoadjuvant chemotherapy; 2) non-mass enhancement (NME) or multiple masses. The patients were separated into two cohorts: the primary cohort $(n=326)$ and the validation cohort $(n=121)$.

\section{MRI Examination}

All MRIs were performed with a $1.5 \mathrm{~T}$ MRI scanner (Magnetom Avanto; Siemens Medical Solutions, Erlangen, Germany) using an 8-channel breast coil with the patient positioned in the center of the magnet in the prone position. Axial dynamic contrast-enhanced images using a 3D T1-weighted volume interpolated body examination (T1W-VIBE) sequence (TR/TE, 4.6/1.5 msec; flip angle, $10^{\circ}$; FOV, $360 \times 360 \mathrm{~mm}^{2}$; matrix, $205 \times 256$; slice thickness, $2 \mathrm{~mm}$ and gap, $0 \mathrm{~mm}$ ) were acquired before and eight dynamic scans after a bolus injection of $0.2 \mathrm{mmol} / \mathrm{kg}$ Gd-DTPA (Omniscan, GE Healthcare, Ireland) at a rate of $2 \mathrm{ml} / \mathrm{s}$, followed by a $20-\mathrm{ml}$ saline flush. In the postprocessing workstation, the region of interest was placed in the enhancement of the largest slice of the tumor to obtain a kinetic curve.

\section{Image interpretation}

According to the BI-RADS [12], morphological and DCE features were independently reviewed by two radiologists (with 8 years and 5 years of experience in breast imaging) without knowledge of the histopathological diagnosis. If the assessments were inconsistent, a consensus was reached by the senior radiologist. The morphological features included tumor size, shape (oval, round or irregular), and margin (circumscribed or non-circumscribed). The DCE features included internal enhancement characteristics (homogeneous or non-homogeneous) and the delayed enhancement features of the kinetic curve (persistent or non-persistent). In addition, age and tumor location were also included.

\section{Construction of the MRI-based nomogram}

Univariate logistic regression analysis was performed to identify the potential MRI features associated with the likelihood of malignancy in patients with breast masses. Subsequently, multivariate logistic regression analysis was conducted to determine the independent predictors. Finally, based on the multivariate logistic regression analysis, the MRI-based nomogram was constructed using the primary cohort, and validated internally by the validation cohort. 


\section{Assessment of MRI-based nomogram performance}

The MRI-based nomogram performance was quantified with respect to the discrimination, calibration ability and clinical utility. The Hosmer-Lemeshow test was used to evaluate the goodness-of-fit of the MRI-based nomogram, and the area under the curve (AUC) value of receiver operating characteristic curve (ROC) was carried out to quantify the discrimination ability of the nomogram. Subsequently, a calibration curve was performed to assess the calibration ability of the nomogram. Decision curve analysis was used to evaluate the clinical utility of the model by calculating the net benefits for a range of threshold probabilities.

\section{Statistical analysis}

Univariate and multivariate logistic regression analyses were performed to identify the independent variables. All data analyses were conducted by using Stata/MP, version 13.0 (StataCorp LP, College Station, TX) and $R$ software version 3.4.1. $P$ value $<0.05$ was considered statistically significant difference.

\section{Results}

\section{Study Population}

Our study population consisted of 447 female patients. The age of included patients ranged from 24 to 78 years, with a mean age of 49 years. The median tumor size was $25 \mathrm{~mm}$. Of the 447 breast masses identified in 447 patients, 375 were malignant and 72 were benign. The baseline characteristics of our study were showed in Table 1.

Table 1. Baseline characteristics of the study population

\begin{tabular}{|c|c|c|c|c|}
\hline \multirow[t]{2}{*}{ Characteristics } & Total cohort & Primary cohort & Validation cohort & \multirow[t]{2}{*}{$P$ value } \\
\hline & No. (\%) & No. $(\%)$ & No. $(\%)$ & \\
\hline No. of patients & 447 & 326 & 121 & \\
\hline Age (years) & & & & 0.792 \\
\hline mean $\pm \mathrm{SD}^{*}$ & $49.6 \pm 10.5$ & $49.3 \pm 10.5$ & $50.4 \pm 10.6$ & \\
\hline$\leq 50$ & $241(53.9)$ & $177(54.3)$ & $64(52.9)$ & \\
\hline$>50$ & $206(46.1)$ & $149(45.7)$ & $57(47.1)$ & \\
\hline Tumor size (mm) & & & & 0.413 \\
\hline Median (IQR) & $25(18,34)$ & $25(18,34)$ & $25(18,33)$ & \\
\hline$\leq 20$ & $160(35.8)$ & $113(34.7)$ & $47(38.8)$ & \\
\hline$>20$ & $287(64.2)$ & $213(65.3)$ & $74(61.2)$ & \\
\hline Tumor location & & & & 0.677 \\
\hline UOQ & $203(45.4)$ & $150(46.0)$ & $53(43.8)$ & \\
\hline Othersa & $244(54.6)$ & $176(54.0)$ & $68(56.2)$ & \\
\hline Mass shape & & & & 0.444 \\
\hline Oval or round & $264(59.1)$ & $189(58.0)$ & $75(62.0)$ & \\
\hline Irregular & $183(40.9)$ & $137(42.0)$ & $46(38.0)$ & \\
\hline Mass margin & & & & 0.137 \\
\hline Non-circumscribed ${ }^{b}$ & $352(78.7)$ & $251(77.0)$ & $101(83.5)$ & \\
\hline Circumscribed & $95(21.3)$ & $75(23.0)$ & $20(16.5)$ & \\
\hline Internal enhancement characteristics & & & & 0.175 \\
\hline Non-homogeneousc & $350(78.3)$ & $250(76.7)$ & $100(82.6)$ & \\
\hline Homogeneous & $97(21.7)$ & $76(23.3)$ & $21(17.4)$ & \\
\hline Kinetics curve & & & & 0.550 \\
\hline Non-persistent & $400(89.5)$ & $290(89.0)$ & $110(90.9)$ & \\
\hline Persistent & $47(10.5)$ & $36(11.0)$ & $11(9.1)$ & \\
\hline BI-RADS category & & & & 0.960 \\
\hline 4 & $206(46.1)$ & $150(46.0)$ & $56(46.3)$ & \\
\hline 5 & $241(53.9)$ & $176(54.0)$ & $65(53.7)$ & \\
\hline Histopathological type & & & & 0.331 \\
\hline Fibroadenoma & $38(8.5)$ & $30(9.2)$ & $8(6.6)$ & \\
\hline Other benign ${ }^{d}$ & $34(7.6)$ & $23(7.1)$ & $11(9.1)$ & \\
\hline IDC & $339(75.8)$ & $243(74.5)$ & $96(79.3)$ & \\
\hline Other malignante & $36(8.1)$ & $30(9.2)$ & $6(5.0)$ & \\
\hline Pathological diagnosis & & & & 0.887 \\
\hline Benign & $72(16.1)$ & $53(16.3)$ & 19 (15.7) & \\
\hline Malignant & $375(83.9)$ & $273(83.7)$ & $102(84.3)$ & \\
\hline
\end{tabular}

Note. *Data are means \pm standard deviation. IQR $=$ Interquartile range, which is the 25th percentile, 75 th percentile. UOQ $=$ upper outer quadrant. aOthers: UIQ $=$ upper inner quadrant, LIQ = lower inner quadrant, LOQ = lower outer quadrant. bNon-circumscribed: speculated, irregular. ${ }^{\mathrm{N}}$ Non-homogeneous: heterogeneous, rim. dOther benign: benign phyllodes tumor 18, papilloma 11, radial scar 5. IDC: invasive ductal carcinoma. eOther malignant: ductal carcinoma in situ 3, papillary carcinoma 6, adenoid cystic carcinoma 2, metaplastic carcinoma 4, mucinous carcinoma 7, invasive lobular carcinoma 14. 


\section{Predictors of malignancy based on MRI findings}

The results of univariate and multivariate logistic regression analyses were illustrated in Table 2. Based on the result of univariate logistic regression analysis, we found that age, tumor size, mass shape, mass margin, internal enhancement characteristics, and kinetic curve were significantly associated with malignancy. According to the result of multivariate logistic regression analysis, five variables were identified as independent predictors in our study, including age, tumor size, mass margin, internal enhancement characteristics and kinetic curve. Typical MRI findings of benign and malignant breast masses were illustrated in Fig. 1.

\section{Development of the MRI-based nomogram}

The multivariate logistic regression analysis identified age, tumor size, mass margin, internal enhancement characteristics and kinetic curve as independent variables (Table 2). The MRI-based nomogram was constructed and presented in Fig. 2. A total score was calculated using only five parameters of age, tumor size, mass margin, internal enhancement characteristics and kinetic curve. Then, the total score can be applied to point out the frequency of malignant tumors for individual patients.

\section{Evaluation of the MRI-based nomogram performance}

The $P$ value of the Hosmer-Lemeshow goodness-of-fit test was 0.532 , which showed the MRI-based nomogram fitted well. The AUC of the MRI-based nomogram in the primary cohort and the validation cohort were 0.940 (95\% CI: 0.911-0.970) and 0.894 (95\% CI, 0.816-0.974), which demonstrated the good discrimination ability of our model (Fig. 3). And the calibration curve of the MRI-based nomogram suggested good agreement between prediction and observation (Fig. 4).

MRI-based nomogram scores were assigned for each independent variable in Table 3. To achieve the maximum Youden index (sensitivity+specificity-1) of

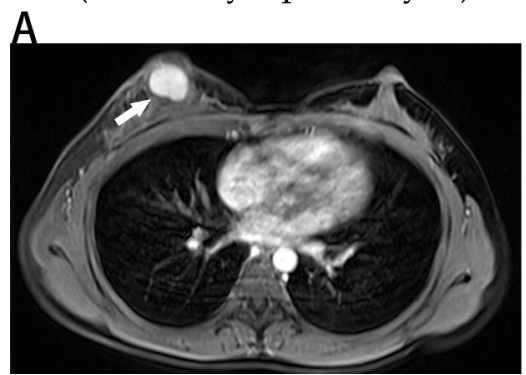

the total MRI-based nomogram scores, 21.704 was identified as the optimal cutoff value of total nomogram scores in the primary cohort. 447 patients were subdivided into the low-risk group (total nomogram scores $\leq 21.704, \mathrm{n}=107$ ) and high-risk group (total nomogram scores $>21.704, n=340$ ) based on the optimal cut-off value. Encouragingly, the high-risk patients had a higher likelihood of harboring malignancy than the low-risk patients (all $P$ $<0.001$ ). The sensitivity, specificity, accuracy, false negative rate and false positive rate of the MRI-based nomogram were $93 \%, 75 \%, 90 \%, 7.5 \%$ and $25 \%$ respectively.

Table 2. Univariate and multivariate logistic regression analyses in the primary cohort

\begin{tabular}{|c|c|c|c|c|}
\hline \multirow[t]{2}{*}{ Variables } & \multicolumn{2}{|l|}{ Univariate analysis } & \multicolumn{2}{|c|}{ Multivariate analysis } \\
\hline & OR $(95 \% \mathrm{CI})$ & $P$ value & OR $(95 \% \mathrm{CI})$ & $P$ value \\
\hline \multicolumn{5}{|l|}{ Age (years) } \\
\hline$\leq 50$ & Referent & & Referent & \\
\hline$>50$ & $4.460(2.154-9.236)$ & $<0.001$ & $\begin{array}{l}2.767 \\
(1.046-7.320)\end{array}$ & 0.040 \\
\hline \multicolumn{5}{|l|}{ Tumor size (mm) } \\
\hline$\leq 20$ & Referent & & Referent & \\
\hline$>20$ & 7.491 (3.847-14.587) & $<0.001$ & $\begin{array}{l}3.579 \\
(1.414-9.061)\end{array}$ & 0.007 \\
\hline \multicolumn{5}{|l|}{ Tumor location } \\
\hline UOQ & Referent & & & \\
\hline Othersa & $1.057(0.586-1.906)$ & 0.853 & & \\
\hline \multicolumn{5}{|l|}{ Mass shape } \\
\hline Oval or round & Referent & & Referent & \\
\hline Irregular & 7.227 (2.991-17.462) & $<0.001$ & $\begin{array}{l}1.414 \\
(0.434-4.603)\end{array}$ & 0.565 \\
\hline \multicolumn{5}{|l|}{ Mass margin } \\
\hline Circumscribed & Referent & & Referent & \\
\hline Non-circumscribed ${ }^{\mathrm{b}}$ & $\begin{array}{l}11.326 \\
(5.854-21.916)\end{array}$ & $<0.001$ & $\begin{array}{l}3.547 \\
(1.328-9.470)\end{array}$ & 0.012 \\
\hline \multicolumn{5}{|l|}{$\begin{array}{l}\text { Internal enhancement } \\
\text { characteristics }\end{array}$} \\
\hline Non-homogeneousc & Referent & & Referent & \\
\hline Homogeneous & $0.037(0.018-0.079)$ & $<0.001$ & $\begin{array}{l}0.116 \\
(0.046-0.292)\end{array}$ & $<0.001$ \\
\hline \multicolumn{5}{|l|}{ Kinetics curve } \\
\hline Persistent & Referent & & Referent & \\
\hline Non-persistent & $\begin{array}{l}25.330 \\
(11.044-58.076)\end{array}$ & $<0.001$ & $\begin{array}{l}7.875 \\
(2.547-24.344)\end{array}$ & $<0.001$ \\
\hline
\end{tabular}

Note. $\mathrm{UOQ}=$ upper outer quadrant. aOthers: UIQ = upper inner quadrant, LIQ = lower inner quadrant, $\mathrm{LOQ}=$ lower outer quadrant. ${ }^{\mathrm{b} N o n-c i r c u m s c r i b e d}$ : speculated, irregular. ${ }^{\mathrm{N}}$ Non-homogeneous: heterogeneous, rim.

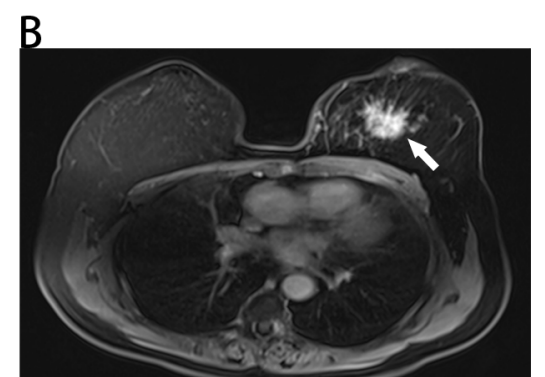

Figure 1. Benign and malignant breast masses on MRI. (A) Benign-looking breast mass of $17 \times 12 \mathrm{~mm}$ (arrow), oval, circumscribed margin and homogeneous enhancement in a 40 -year-old woman. Pathological diagnosis: fibroadenoma. (B) Malignant-looking breast mass of $36 \times 32 \mathrm{~mm}$ (arrow), irregular, non-circumscribed margin and heterogeneous enhancement in a 60-year-old woman. Pathological diagnosis: invasive ductal carcinoma. 
Points

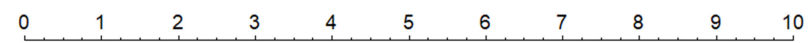

Age

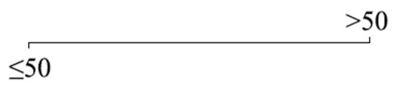

Tumor size

$\leq 2 \quad>20$

Mass margin

Circumscribed

Internal enhancement

Non-homogeneous

characteristics

Homogeneous

Kinetic curve

$$
\text { Persistent }
$$

Non-persistent

Total Points

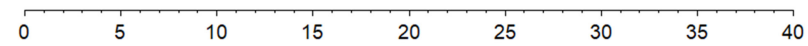

Probability

$$
\begin{array}{lllllllllllll}
0.05 & 0.1 & 0.2 & 0.3 & 0.4 & 0.5 & 0.6 & 0.7 & 0.8 & 0.9 & 0.95 & 0.98 & 0.99
\end{array}
$$

Figure 2. The MRI-based nomogram to predict the risk of malignancy in patients with breast masses based on MRI findings.
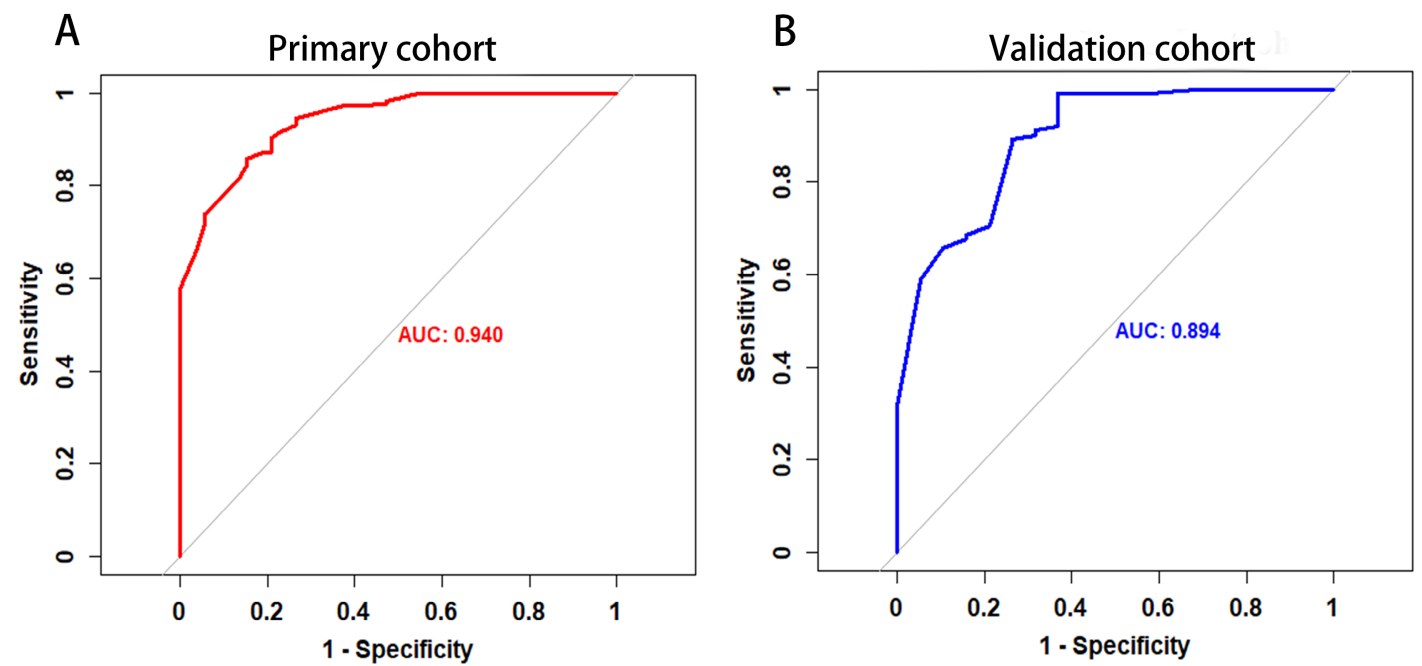

Figure 3. The ROC curve of MRI-based nomogram in the primary cohort and the validation cohort.

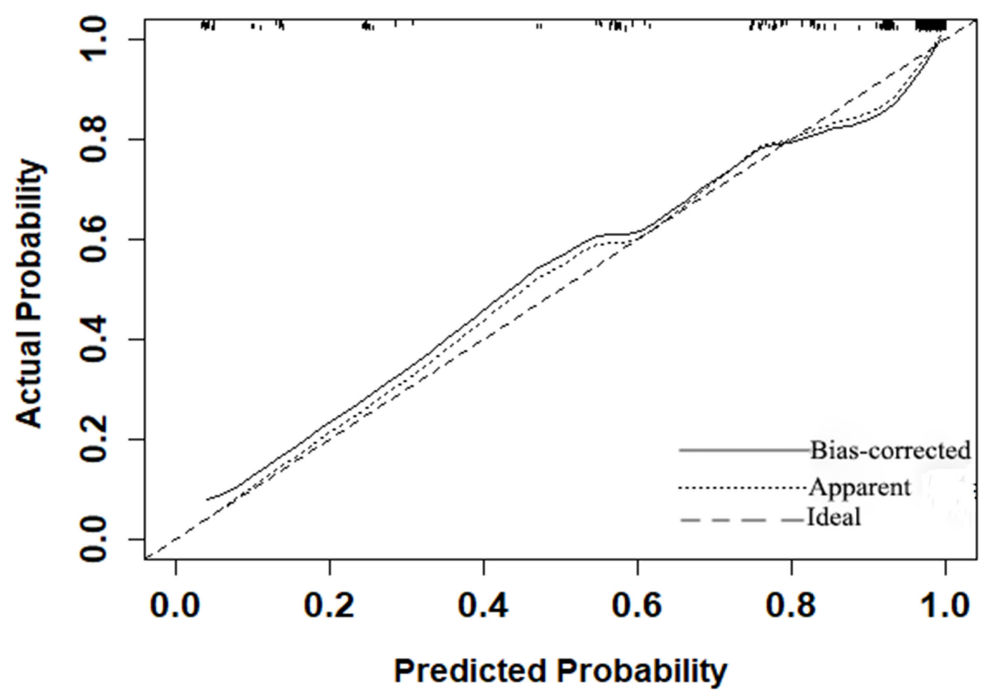

Figure 4. Calibration curve of the MRI-based nomogram to predict the risk of malignancy in patients with breast masses. 


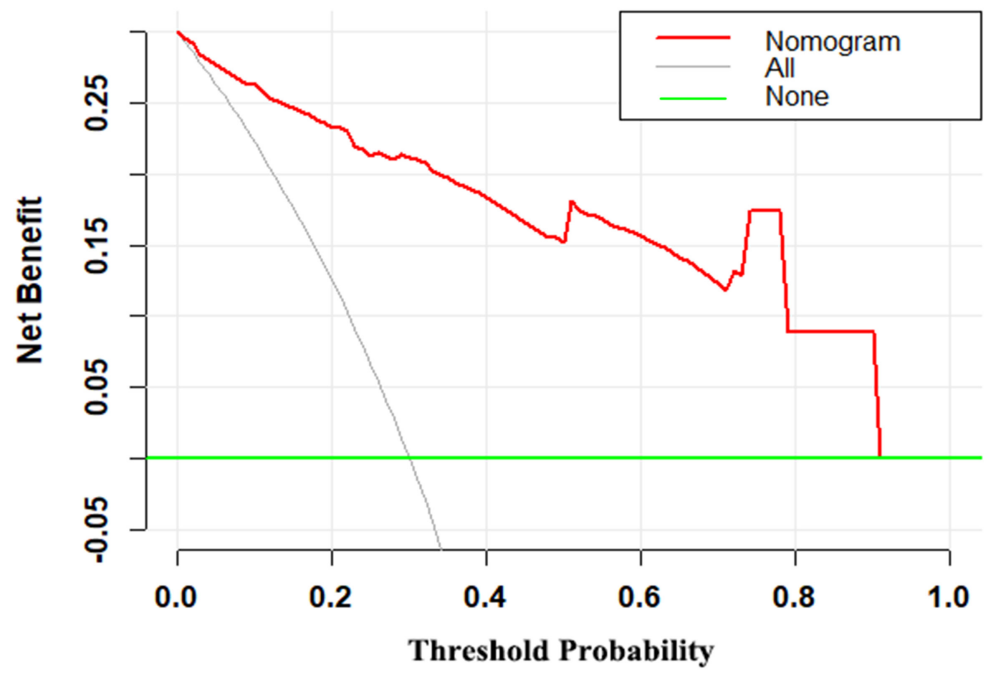

Figure 5. Decision curve for the MRI-based nomogram. The red line represents the MRI-based nomogram. The gray line represents the hypothesis that all patients had malignant mass. The green line represents the hypothesis that all patients had benign mass. The $\mathrm{x}$-axis represents the threshold probability. The $y$-axis represents the net benefit.

Table 3 MRI-based nomogram score for each variable

\begin{tabular}{ll}
\hline Variables & Nomogram Score \\
\hline Age (years) & 0 \\
$\leq 50$ & 4.430 \\
$>50$ & 0 \\
Tumor size (mm) & 5.835 \\
$\leq 20$ & \\
$>20$ & 0 \\
Mass margin & 6.218 \\
Circumscribed & 0 \\
Non-circumscribeda & 10 \\
Internal enhancement characteristics & \\
Homogeneous & \\
Non-homogeneous & \\
Kinetics curve & 0 \\
Persistent & 9.301 \\
Non-Persistent & \\
Risk stratification & $>21.704$ \\
Low-risk & $>21.704$ \\
High-risk & ing
\end{tabular}

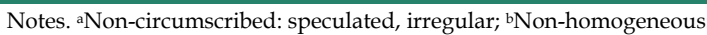
heterogeneous, rim

\section{Clinical utility of MRI-based nomogram}

The decision curve demonstrated that the MRI-based nomogram had good clinical utility across the wider range of threshold probability (Fig 5).

\section{Discussion}

In this study, we have first proposed and established a diagnostic MRI-based nomogram which could individually predict the likelihood of malignancy in patients with breast masses. Nomogram has an ability to provide an individual risk of a clinical event for personalized treatment [14]. The MRI-based nomogram incorporated patients age, tumor size and three MRI parameters (mass margin, internal enhancement characteristics and kinetic curve). It is generally known that the ROC-AUC of $0.7-0.8$ is deemed favorable and the ROC-AUC of $0.81-0.90$ is excellent. Thus, the discrimination ability of the MRI-based nomogram was outstanding (ROC-AUC $=0.940$ ). Additionally, the HosmerLemeshow goodness-of-fit test yielded the $P$ value of 0.532, showing a good fit to the MRI-based nomogram. Besides, the calibration curve demonstrated good calibration ability of the MRI-based nomogram. The decision curve analysis has been recommended to assess the potential clinical usefulness of model [15-20]. This important method gains novel insight into clinical consequences based on threshold probability, from which the net benefit can be derived $[14,15,21]$. The decision curve demonstrated that the MRI-based nomogram had better clinical utility with the wider range of threshold probabilities. Furthermore, the MRI-based model also can make effective risk stratification in the diagnosis setting. Therefore, the MRI-based nomogram performed well for preoperative differential diagnosis between benign and malignant breast masses, with favorable clinical utility, risk stratification, discrimination, and calibration ability.

Our study developed a non-invasive preoperative nomogram for predicting the risk of malignancy in patients with breast masses based on MRI findings. Three MRI parameters were adopted as independent indicators to estimate the probability of malignancy in the MRI-based nomogram, including mass margin, internal enhancement characteristics and kinetic curve. Similarly, several prior studies had revealed breast masses with irregular shape, spiculated margin and plateau or washout patterns curve had greater probability of malignancy $[11,22-25]$. Mass shape does appear to be a potential 
independent variable of malignancy. Nevertheless, in the present study, mass shape was confirmed as an indicator of malignancy in a univariate analysis but lost the statistical significant when added to the multivariate analysis, which was in line with previous study [22]. Multiple previous studies have combined the morphologic and DCE features to evaluate the likelihood of malignancy for breast MRI masses based on BI-RADS MRI descriptors [22,23]. However, these reports only identified which BI-RADS descriptors were associated with malignancy, cannot provide objective individualized risk prediction in patients with breast mass. Thereby, providing individualized risk prediction by a practical tool remained absence.

The MRI-based nomogram has some clinical significance. First, the MRI-based nomogram only includes five readily available preoperative parameters: age, tumor size, mass margin, internal enhancement characteristics and kinetic curve. Thus, the MRI-based nomogram can easily calculate the risk of malignancy for individual patient. Second, when two radiologists have inconsistent diagnosis of breast lesions, the MRI-based nomogram can be used as a user-friendly tool to reduce inter-observer variability. Third, based on the MRI-based nomogram, breast masses with a low likelihood of malignancy may be qualified for short-interval follow-up rather than breast biopsy. Fourth, the MRI-based nomogram can also provide effective communication among radiologists, oncologists, and patients.

There are some limitations in the study. First, most cases were malignant, but the result was significant. Second, this study was conducted at one institution and was a retrospective study. the multicenter validation studies should be performed before its clinical application in the future. Third, future investigations are needed to establish a preoperative nomogram for predicting the risk of malignancy in patients with non-mass enhancement. Fourth, the initial enhancement feature of the kinetic curve was not included in our study.

\section{Conclusion}

In summary, the proposed MRI-based nomogram, a preoperative prediction tool which incorporates the morphological and DCE features, suggests favorable predictive accuracy for malignancy in patients with breast masses and makes effective risk stratification in the diagnosis setting. The MRI-based nomogram may be used as an important decision-making tool in differentiating malignant from benign breast masses and reducing unnecessary breast biopsy in the low-risk patients.

\section{Abbreviations}

DCE: dynamic contrast-enhanced; MRI: magnetic resonance imaging; BI-RADS: Breast Imaging Reporting and Data System; ACR: American College of Radiology; NME: non-mass enhancement; AUC: area under the curve; ROC: receiver operating characteristic curve

\section{Acknowledgements}

This study has received funding by the National Natural Science Foundation of China (81972467, 81472731, 82002928), the grants from Key Laboratory of Malignant Tumor Molecular Mechanism and Translational Medicine of Guangzhou Bureau of Science and Information Technology (Grant [2013]163), from the Key Laboratory of Malignant Tumor Gene Regulation and Target Therapy of Guangdong Higher Education Institutes (KLB09001), from Guangdong Science and Technology Department (2015B050501004), and from the Doctor Launch Fund of Guangdong Provincial People's hospital (2020bq11).

\section{Competing Interests}

The authors have declared that no competing interest exists.

\section{References}

1. DeSantis CE, Ma J, Gaudet MM, et al. Breast cancer statistics, 2019. CA Cancer J Clin. 2019;69:438-451.

2. Marino MA, Helbich T, Baltzer P, Pinker-Domenig K. Multiparametric MRI of the breast: A review. J Magn Reson Imaging. 2018;47:301-315.

3. Sardanelli F, Giuseppetti GM, Panizza P, et al. Sensitivity of MRI versus mammography for detecting foci of multifocal, multicentric breast cancer in Fatty and dense breasts using the whole-breast pathologic examination as a gold standard. AJR Am J Roentgenol. 2004;183:1149-1157.

4. Kristoffersen Wiberg M, Aspelin P, Sylvan M, Boné B. Comparison of lesion size estimated by dynamic MR imaging, mammography and histopathology in breast neoplasms. Eur Radiol. 2003;13:1207-1212.

5. Kriege M, Brekelmans CT, Boetes C, et al. Efficacy of MRI and mammography for breast-cancer screening in women with a familial or genetic predisposition. N Engl J Med. 2004;351:427-437.

6. Leach MO, Boggis CR, Dixon AK, et al. Screening with magnetic resonance imaging and mammography of a UK population at high familial risk of breast cancer: a prospective multicentre cohort study (MARIBS). Lancet. 2005;365:1769-1778.

7. Kuhl CK, Schrading S, Leutner CC, et al. Mammography, breast ultrasound, and magnetic resonance imaging for surveillance of women at high familial risk for breast cancer. J Clin Oncol. 2005;23:8469-8476.

8. Orel SG, Schnall MD, LiVolsi VA, Troupin RH. Suspicious breast lesions: MR imaging with radiologic-pathologic correlation. Radiology. 1994;190:485-493.

9. Bluemke DA, Gatsonis CA, Chen $\mathrm{MH}$, et al. Magnetic resonance imaging of the breast prior to biopsy. JAMA. 2004;292:2735-2742.

10. Kuhl CK, Mielcareck P, Klaschik S, et al. Dynamic breast MR imaging: are signal intensity time course data useful for differential diagnosis of enhancing lesions? Radiology. 1999;211:101-110.

11. Wedegartner U, Bick U, Wortler K, Rummeny E, Bongartz G. Differentiation between benign and malignant findings on MR-mammography: usefulness of morphological criteria. Eur Radiol. 2001;11:1645-1650.

12. Morris EA, Comstock C, Lee C, et al. ACR BI-RADS Magnetic Resonance Imaging. In: ACR BI-RADS Atlas, Breast Imaging Reporting and Data System. Reston, VA:American College of Radiology;2013:11-173.

13. Iasonos A, Schrag D, Raj GV, Panageas KS. How to build and interpret a nomogram for cancer prognosis. J Clin Oncol. 2008;26:1364-1370.

14. Balachandran VP, Gonen M, Smith JJ, DeMatteo RP. Nomograms in oncology: more than meets the eye. Lancet Oncol. 2015;16:e173-e180.

15. Vickers AJ, Elkin EB. Decision curve analysis: a novel method for evaluating prediction models. Med Decis Making. 2006;26:565-574. 
16. Kerr KF, Brown MD, Zhu K, Janes H. Assessing the Clinical Impact of Risk Prediction Models With Decision Curves: Guidance for Correct Interpretation and Appropriate Use. J Clin Oncol. 2016;34:2534-2540.

17. Vickers AJ. Decision analysis for the evaluation of diagnostic tests, prediction models and molecular markers. Am Stat. 2008;62:314-320

18. Vickers AJ, Cronin AM, Elkin EB, Gonen M. Extensions to decision curve analysis, a novel method for evaluating diagnostic tests, prediction models and molecular markers. BMC Med Inform Decis Mak. 2008;8:53.

19. Vickers AJ, Van Calster B, Steyerberg EW. Net benefit approaches to the evaluation of prediction models, molecular markers, and diagnostic tests. BMJ. 2016;352:16.

20. Fitzgerald M, Saville BR, Lewis RJ. Decision curve analysis. JAMA. 2015;313:409-410.

21. Collins GS, Reitsma JB, Altman DG, Moons KG. Transparent reporting of a multivariable prediction model for individual prognosis or diagnosis (TRIPOD): the TRIPOD statement. BMJ. 2015;350:g7594.

22. Gutierrez RL, DeMartini WB, Eby PR, Kurland BF, Peacock S, Lehman CD. BI-RADS Lesion Characteristics Predict Likelihood of Malignancy in Breast MRI for Masses But Not for Nonmasslike Enhancement. AJR Am J Roentgenol. 2009;193:994-1000.

23. Mahoney MC, Gatsonis C, Hanna L, DeMartini WB, Lehman C. Positive predictive value of BI-RADS MR imaging. Radiology. 2012;264:51-58.

24. Agrawal G, Su MY, Nalcioglu O, Feig SA, Chen JH. Significance of breast lesion descriptors in the ACR BI-RADS MRI lexicon. Cancer. 2009;115:1363-1380.

25. Schnall MD, Blume J, Bluemke DA, et al. Diagnostic architectural and dynamic features at breast MR imaging: multicenter study. Radiology. 2006;238:42-53. 